\title{
Systematische Entwicklung von Dienstleistungen - Chancen für Beschäftigung und Wachstum
}

\author{
Walter Ganz/Klaus-Peter Fähnrich \\ Thomas Meiren/Kyrill Meyer
}

\begin{abstract}
Die systematische Entwicklung neuer Dienstleistungen spielte in Praxis und Wissenschaft lange Zeit keine Rolle. Erst in der zweiten Hälfte der 1990er Jahre entstand „Service Engineering“als ein neues Forschungsgebiet, welches sich mit der systematischen Entwicklung und Gestaltung von Dienstleistungen befasst und dabei versucht, bestehendes Engineering-Know-how effizient für Dienstleistungen zu nutzen. Mit diesem, insbesondere in Deutschland vorangetriebenen systematischen Ansatz zur Entwicklung von Dienstleistungen verbindet sich die Chance, „schlummernde Potenziale“ in Dienstleistungsarbeiten wie auch neue Beschäftigungschancen zu erschließen und die Qualität von Dienstleistungen zu verbessern. Vor allem deshalb, weil dieser Ansatz ganz bewusst neben der Anbieter- auch die Nutzerperspektive berücksichtigt.
\end{abstract}

\section{Dienstleistungen im Kon- text einer zunehmenden Innovationsdynamik}

Mit der wachsenden Bedeutung des Dienstleistungssektors hat auch der Wettbewerb in vielen Dienstleistungsmärkten in den letzten beiden Jahrzehnten deutlich zugenommen. Ehemals träge Märkte sind dynamischer geworden, es sind neue Akteure hinzugekommen (United Nations Economic Commission for Europe 2011; OECD 2005).

Vor diesem Hintergrund sind Dienstleistungsunternehmen häufig nicht mehr in der Lage, sich lediglich durch Kosten-, Image- oder Qualitätsvorteile zu differenzieren. Vielmehr entwickelt sich die Abgrenzung von Wettbewerbern durch innovative Dienstleistungsangebote $\mathrm{zu}$ einem entscheidenden Alleinstellungsmerkmal. Die wesentliche Herausforderung für die Unternehmen besteht darin, vor allem kontinuierlich verbesserte und neue Dienstleistungen auf dem Markt anzubieten, um im Wettbewerb beständig einen Schritt voraus zu sein und gleichzeitig den Bedürfnissen und Erwartungen der Kunden zu entsprechen.

Allerdings zeigt sich, dass viele Unternehmen hierfür kaum gewappnet sind. Es fehlt an geeigneten Strukturen und Prozessen: sowohl für die effiziente Entwick- lung neuer Dienstleistungen als auch für deren Positionierung auf dem Markt. So zeigt eine aktuelle Studie unter 791 europäischen Unternehmen, dass $45 \%$ aller neuen Dienstleistungen bereits im ersten Jahr ihrer Markteinführung scheitern (Edvardsson et al. 2010). Darüber hinaus fehlen noch immer geeignete Methoden und Werkzeuge für die Planung und Umsetzung neuer Dienstleistungen. Sehr oft beginnen die Schwierigkeiten schon damit, dass viele Unternehmen nicht wissen, wie sie ihre Dienstleistungen beschreiben sollen, wie Prozesse zu definieren und Ressourcen zu planen sind (Bullinger et al. 2003).

Für die Praxisakteure ist die Entwicklung neuer Dienstleistungen mittlerweile ein höchst relevantes Thema. ${ }^{1}$ Die Forschung jedoch hat sich der Entwicklung neuer Dienstleistungen erst in den letzten Jahren intensiver zugewandt. Obwohl unter der Überschrift „New Service Development" bereits in den 1980er Jahren erste anglo-amerikanische Arbeiten Eingang in die Forschungsliteratur fanden, geschah dies zunächst auf einem eher rudimentären Level. So kam beispielsweise Bowers nach umfangreichen Recherchen zu dem Schluss: "The single most compelling criticism of the new service development literature is the lack of thereof" (Bowers 1985, S. 42). Die damalige Forschung konzentrierte sich besonders auf grundlegende Rahmenbedingungen, Erfolgsfaktoren und Hindernisse für die Entwicklung neu- er Dienstleistungen, lieferte aber nur sehr selten konkrete Instrumente und Methoden, die in der Praxis angewendet werden

\footnotetext{
1 Das zeigen die Ergebnisse der internationalen Expertenbefragungen in Spath/Ganz (2008) sowie Ganz/Meiren (2002).
}

Walter Ganz, MA, Institutsdirektor am Fraunhofer-Institut für Arbeitswirtschaft und Organisation (IAO), Stuttgart. Arbeitsschwerpunkte: Dienstleistungsmanagement, integrierte Personal- und Organisationsentwicklung.

e-mail:walter.ganz@iao.fraunhofer.de Klaus-Peter Fähnrich, Prof. Dr.-Ing. habil., Universität Leipzig, Institut für Informatik, Betriebliche Informationssysteme. Arbeitsschwerpunkte: Service Engineering, Dienstleistungsinformatik, Softwaretechnik. e-mail: faehnrich@informatik.uni-leipzig.de Thomas Meiren, Dipl.-Wirtschaftsing., Leiter des Competence Teams "Dienstleistungsentwicklung" am IAO. Arbeitsschwerpunkte: Service Engineering, Dienstleistungsorganisation, Dienstleistungs marketing.

e-mail: thomas.meiren@iao.fraunhofer.de Kyrill Meyer, Dr., Leiter der kooperativen Arbeitsgruppe Service Science \& Technology am Institut für Informatik der Universität Leipzig.

Arbeitsschwerpunkte: Service Engineering, technologiegestütztes Innovationsmanagement, IT-basierte Dienstleistungen. e-mail: meyer@informatik.uni-leipzig.de 
konnten. Seit einigen Jahren, zeitgleich mit der steigenden praktischen Relevanz der Thematik, hat sich diese Situation jedoch geändert. Betrachtet man die gegenwärtigen Forschungsaktivitäten, die wachsende Zahl von Publikationen, so sieht man, dass dem Thema Dienstleistungsentwicklung inzwischen erheblich mehr Aufmerksamkeit zuteil wird (Edvardsson et al. 2000, 2006; Fitzsimmons/Fitzsimmons 2000).

Im Gegensatz zur stark marketingorientierten Herangehensweise der ersten amerikanischen Arbeiten näherte man sich in Deutschland dem Thema Dienstleistungsentwicklung eher aus „Engineering-Sicht", d. h. man begann, vorhandenes Know-how aus dem Bereich der konventionellen Produkt- und Softwareentwicklung $\mathrm{zu}$ analysieren, $\mathrm{zu}$ modifizieren und auf Dienstleistungen zu übertragen. „Service Engineering“"kann somit als die systematische Entwicklung und Gestaltung von Dienstleistungen unter Verwendung geeigneter Modelle, Methoden und Werkzeuge verstanden werden. Die Grundidee des Service Engineering ist die Verwendung eines stärker technisch-methodischen Ansatzes und der Versuch, das vorhandene Know-how im Bereich der traditionellen Ingenieurwissenschaften effizient zu nutzen, um innovative Dienstleistungen zu entwickeln. Von besonderem Interesse ist dabei der Transfer von Ingenieurwissen hin zu Dienstleistungen, nicht nur aus dem Produkt- oder Software-Engineering, sondern auch von „weicheren“ Ingenieurdisziplinen, wie dem Industrial Engineering, dem Business Process Engineering und der sozio-technischen Systemgestaltung.

Hintergrund der Entwicklung dieses Ansatzes war jedoch ebenfalls, dass ein Erfolgsfaktor des deutschen Innovationsmodells der „flexiblen Qualitätsproduktion“ der Industrie in der Kompetenz der Ingenieure, Meister und Facharbeiter gesehen wird. Diese Idee bzw. dieses Innovationsmodell auch für die Entwicklung und Erbringung von Dienstleistungen zu nutzen, scheint auch für einen deutschen Pfad in die Dienstleistungsgesellschaft hilfreich zu sein. Man muss allerdings die spezifischen Besonderheiten von Dienstleistungen berücksichtigen, wie beispielsweise, dass Kunden, Klienten, Patienten, Gäste und Beratungssuchende aktiver Bestandteil des Wertschöpfungsprozesses sind. Das aber heißt: Das Wissen über die Besonderheiten von Dienstleistungen setzt der Übertragbarkeit des deutschen industriellen Inno- vationsmodells auch Grenzen.

Gegenwärtig lässt sich eine Diskussion zur Entwicklung eines Leitbildes Dienstleistungsfacharbeit beobachten (Bienzeisler/Hermann 2010). Inzwischen hat dieser Ansatz der systematischen Entwicklung von Dienstleistungen auch eine disziplinäre Erweiterung in Richtung Einbindung betriebswirtschaftlichen und sozialwissenschaftlichen Know-hows erfahren und einen stärkeren ganzheitlichen Anspruch, weil insbesondere bestimmte Dienstleistungen dialogisch-interaktive Arbeit sind (Hacker 2009). Festhalten lässt sich jedoch, dass in den letzten Jahren auf dem Gebiet des Service Engineering eine Fülle von Erfahrungen zusammengetragen wurde (Ernst 2011; Bullinger/Scheer 2006; Meiren/Barth 2002).

Grundsätzlich ist der Ansatz des Service Engineering für ein breites Spektrum von Dienstleistungen geeignet, insbesondere wenn es um die Entwicklung von neuen innovativen Dienstleistungsprodukten, aber auch wenn es um die Gestaltung effizienter Leistungserstellungsprozesse geht. Zugleich zeigt sich, dass Know-how aus den sozialwissenschaftlichen Disziplinen notwendig ist, wenn es sich insbesondere um interaktions- und wissensintensive Dienstleistungen handelt, weil die Gestaltung dialogisch-interaktiver Dienstleistungsarbeit am oder mit anderen Menschen, zum Beispiel im Gesundheitsbereich, besondere Herausforderungen stellt. Doch trotz einer gewissen Komplexität sind die bis dato entwickelten Gestaltungsansätze keineswegs nur für größere Unternehmen interessant, wie Beispiele aus der Praxis inzwischen zeigen: „Service Engineeringdie systematische Entwicklung von Dienstleistungen - ist ein sperriger Begriff, doch auch wiederum das Werkzeug für Unternehmen jeder Größenklasse zur Erzielung erstklassiger interner Prozesse und exzellenter Produkte" (Zühlke-Robinet 2009). Hinzu kommt, und das scheint besonders bedeutsam, dass sich inzwischen feststellen lässt, dass Unternehmen zunehmend Mitarbeiter suchen, die Kompetenzen in der Entwicklung und Gestaltung von Dienstleistungen haben, und das gilt nicht nur für Akademiker, sondern auch für Personen mit mittleren Qualifikationen.

\section{2 \\ Ausgewählte Forschungs- bereiche des Service Engineering}

Wie bereits erwähnt, versteht sich Service Engineering als eine sozio-technische Disziplin, die sich im Kern mit der systematischen Entwicklung und Gestaltung von Dienstleistungsprodukten befasst und dabei alle Phasen des Prozesses von Forschung und Entwicklung (F\&E), d.h. von der ersten Idee bis hin zur Konzeption, Umsetzung und Markteinführung neuer Dienstleistungen, berücksichtigt. Im Fokus stehen dabei Modelle, Methoden und Werkzeuge, um die Entwicklung des „F\&E-Objekts Dienstleistung“ zu unterstützen, aber auch Lösungen im Sinne eines geeigneten F\&E-Managements für Dienstleistungen werden untersucht. $A b$ bildung 1 zeigt die Struktur des Arbeitsbereichs in vereinfachter Form.

\subsection{MODELLE, METHODEN UND WERKZEUGE FÜR DIE ENTWICK- LUNG NEUER DIENSTLEISTUNGEN}

Anfängliche Arbeiten fokussierten stark auf die Bereitstellung von Prozessmodellen und -methoden für die Entwicklung

\section{Abb. 1: Service Engineering}

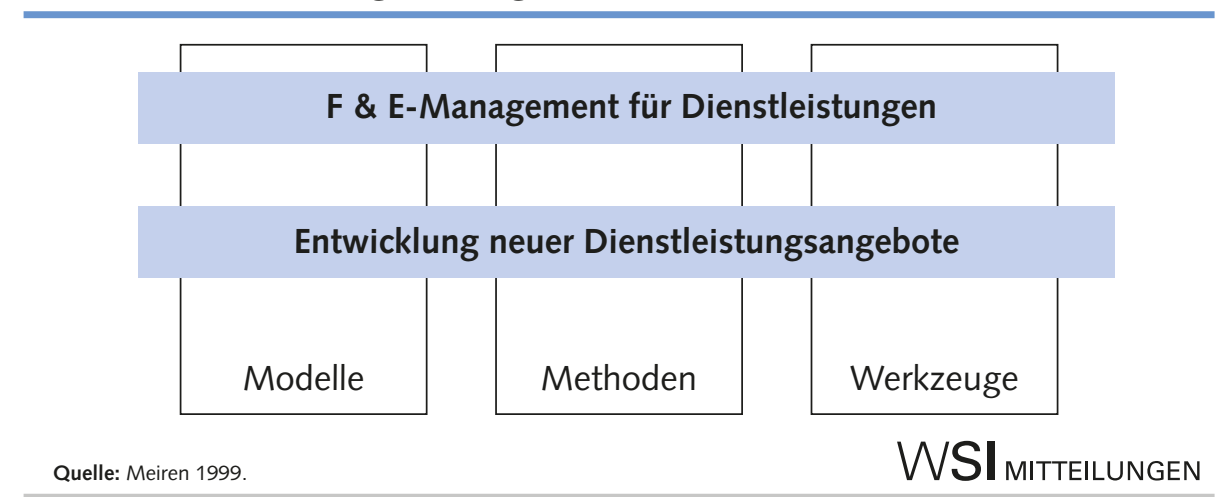




\section{Abb. 2: Beispiel eines Dienstleistungsentwicklungsprozesses}

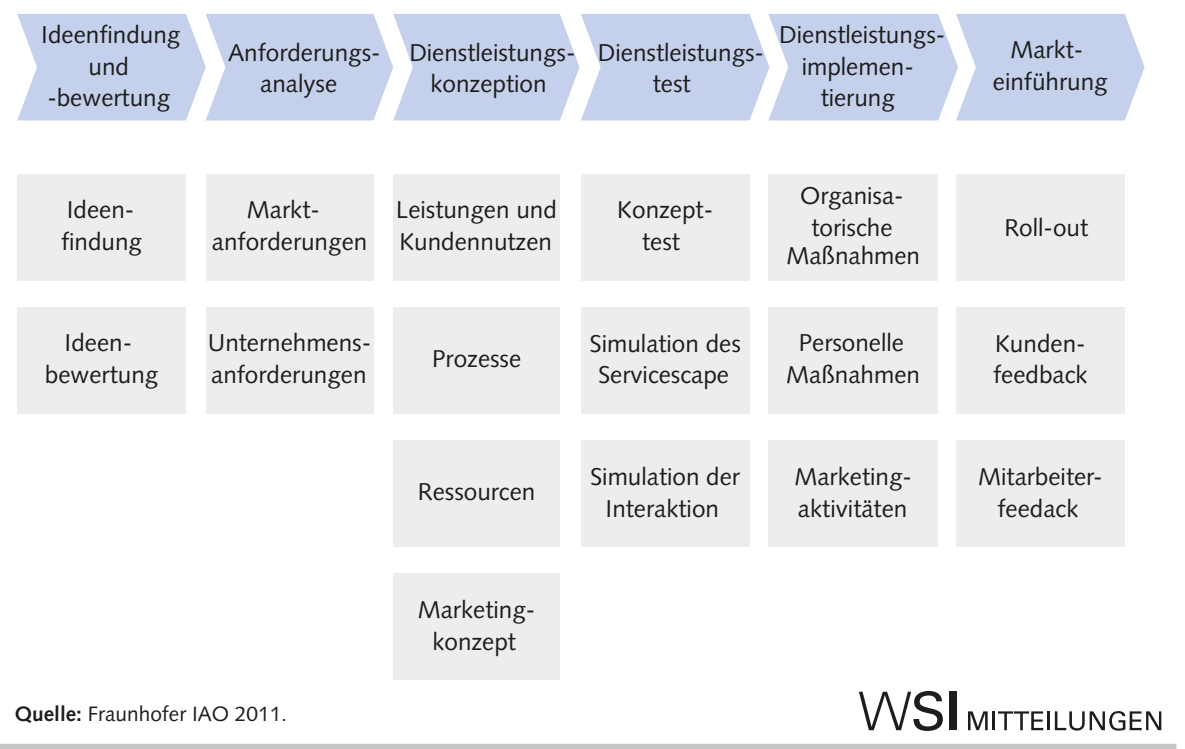

von Dienstleistungen. Dies beinhaltete die Beschreibung der Entwicklungsprozesse - oft analog zur traditionellen Produktund Softwareentwicklung. Beispielsweise wurden zahlreiche Vorgehensmodelle entwickelt, die der detaillierteren Dokumentation von Projektabläufen, Projektstrukturen und Projektverantwortlichkeiten und damit der Planung, Steuerung und Überwachung von F\&E-Projekten für Dienstleistungen dienten. Hervorzuheben ist hierbei, dass bereits die ersten Modelle die Besonderheiten von Dienstleistungen wie etwa Kundenintegration und -interaktion berücksichtigten.

Bis heute wurde eine Vielzahl an Vorgehensweisen für die Entwicklung von Dienstleistungen vorgestellt (Bullinger/ Scheer 2006). Allerdings sind die für die Dienstleistungsentwicklung verwendeten Prozessmodelle noch immer meist einfacher Natur (Abbildung 2). Viele folgen einem sequenziellen Phasenmodell und bieten nur wenige Möglichkeiten zur Konfiguration, etwa wenn es um die Anpassung an unterschiedliche Typen von Dienstleistungen geht. Neuere Modelle differenzieren hier stärker und ermöglichen außerdem die parallele Entwicklung von Dienstleistungen und unterstützender Software durch ein Software-Service-CoDesign (Meyer 2009).

In der Praxis erfordert die Entwicklung erfolgreicher Dienstleistungen operative Unterstützung in Form von geeigneten Methoden und Werkzeugen (Bullinger et al. 2003). Ein Blick in die Literatur zeigt, dass bisher nur sehr wenige dienstleistungsspezifische Instrumente (z. B. Service Blueprinting) bereitstehen (Burger et al. 2010). Stattdessen werden in der Praxis vielfach bewährte Methoden und Werkzeuge aus der Produkt- und Softwareentwicklung (z. B. TRIZ, QFD, SADT, FMEA, $(\mathrm{UML})^{2}$ übertragen. Doch obwohl hier ein reichhaltiger Bestand an Methoden und Werkzeugen vorhanden ist, können diese nicht wahllos für Dienstleistungen genutzt werden, da Dienstleistungen einen hohen Grad an Intangibilität aufweisen und zudem die Gestaltung der Interaktion von Kunden und Mitarbeitern häufig sehr entscheidend für die erfolgreiche Umsetzung von neuen Dienstleistungen ist. Eine unreflektierte Methodenübertragung, die diesen Besonderheiten nicht gerecht wird, ist demzufolge schnell zum Scheitern verurteilt (Fähnrich/Meiren 2007).

Und ein weiterer Unterschied zur Produkt- und Softwareentwicklung ist hervorzuheben: Obwohl zunehmend mehr Methoden und Werkzeuge für die systematische Entwicklung von Dienstleistungen entwickelt und erprobt werden, gibt es noch immer keine nahtlose Verbindung zwischen ihnen und es fehlt an integrierter Software-Unterstützung, die mit Computer-Aided Design-Tools (CAD) in der Produktentwicklung oder Computer-Aided Software Engineering Tools (CASE) in der Softwareentwicklung vergleichbar wäre. Zwar wurden inzwischen erste Konzepte für Software-Plattformen entwickelt (Arai/Shimomura 2004) und erste Prototy- pen realisiert (Fähnrich/van Husen 2008) - von einer praktischen Nutzbarkeit sind diese jedoch noch weit entfernt.

\subsection{F\&E-MANAGEMENT FÜR DIENSTLEISTUNGEN}

Moderne Engineering-Ansätze befassen sich nicht allein mit dem Entwicklungsobjekt, sondern meist auch mit dem kompletten Entwicklungssystem, mit dem Ziel, umfassende Lösungen für Forschung und Entwicklung zu erzeugen. Dies gilt auch für die Entwicklung und Gestaltung von Dienstleistungen, denn für viele Unternehmen sind die anstehenden Aufgaben völlig neu, und die Implementierung und Integration der Dienstleistungsentwicklung in einem Unternehmen wirft eine ganze Reihe von Fragen auf: Welche Bereiche eines Unternehmens müssen bei der Entwicklung neuer Dienstleistungen integriert werden? Welche organisatorischen Konzepte (z.B. eigene F\&E-Abteilungen für Dienstleistungen, versus dezentrale, virtuelle Strukturen) sind geeignet? Wie kann man Informationsflüsse und Wissenstransfer organisieren? Wie lässt sich der Entwicklungsprozess steuern? Wie schafft es ein Unternehmen, kontinuierlich neue, wettbewerbsfähige Dienstleistungen auf den Markt zu bringen?

Vor diesem Hintergrund konzentriert sich eine zunehmende Zahl an Forschungsarbeiten auf die organisatorische Verankerung der Dienstleistungsentwicklung in Unternehmen und versucht, geeignete Organisationsformen zu identifizieren wie etwa die Einrichtung von spezialisierten Fachabteilungen -, die am besten für diese Aufgabe geeignet sind. Ein weiteres Anwendungsfeld in diesem Kontext sind Fragestellungen der Internationalisierung von Dienstleistungen. Hierbei geht es nicht nur um den „klassischen Export“, sondern auch um die Frage, wie sich die Unternehmen in internationalen Wertschöpfungsnetzwerken platzieren können. Um auf dem internationalen Parkett Dienstleistungen erfolgreich vermarkten zu können, sind Unternehmen, welche der Bedeutung und den Anforderungen des internationalen Dienstleistungsgeschäfts

\footnotetext{
2 TRIZ= Theorie des erfinderischen Problemlösens (russ. теория решения иззобретательских задач); QFD = Quality Function Deployment; SADT= Structured Analysis and Design Technique; FMEA = Fehler-Möglichkeits- und Einflussanalyse; $U M L=$ Unified Modeling Language.
} 
gerecht werden wollen, vielfältigen neuartigen Herausforderungen ausgesetzt. Der Ansatz des Service Engineering bietet erprobte und praktikable Handlungskonzepte als Orientierung und Anleitung für einen langfristigen Erfolg im internationalen Dienstleistungsmarkt (Meyer et al. 2008).

\subsection{MODELLIERUNG UND SIMU- LATION VON DIENSTLEISTUNGEN}

Während eine relativ große Anzahl an akademischen Arbeiten auf Begriffsbestimmungen, Merkmale und Typologien von Dienstleistungen zugeschnitten war, fällt auf, dass die Entwicklung von grundlegenden Modellen für Dienstleistungen vernachlässigt wurde. Die Gestaltung von Modellen ist jedoch ein wichtiger Bestandteil für die Entwicklung theoretischer Grundlagen, aber auch Voraussetzung für praxisbezogene Aktivitäten wie die Modellierung und Simulation von Dienstleistungen. In Bezug auf Letzteres existieren nur sehr wenige dienstleistungsspezifische Methoden; die bisher genutzten Verfahren wurden meist aus dem Business Process Engineering auf den Dienstleistungssektor übertragen, wobei die für Dienstleistungen so wichtigen „weichen Faktoren“ wie etwa Interaktion, Verhalten und Emotion in der Regel nicht abgebildet werden können (Meyer/Münter 2010).

Weitere Forschungsarbeiten im Bereich der Dienstleistungsmodellierung beschäftigen sich mit der Fragestellung einer formalen Beschreibung von Dienstleistungen bzw. komplexen Dienstleistungssystemen. Das Ziel ist, diese sprachlich adäquat zu beschreiben und darauf aufbauende informationstechnische Werkzeuge zu entwickeln, die eine integrierte, lebenszyklusorientierte und ganzheitliche Erfassung und Gestaltung aller leistungsrelevanten Elemente einer Dienstleistung ermöglichen. Erste Konzepte wurden bereits für Dienstleistungsproduktkomponenten, Ressourcen und Prozesse erarbeitet (Böttcher 2008 und Abbildung 3). Aufbauend darauf kann eine Modellbildung erfolgen.

Das materielle Umfeld, in dem sich die Erbringung einer Dienstleistung abspielt, wird in der Literatur auch als „Servicescape" bezeichnet. Hierbei wurden wichtige Elemente und Einflussfaktoren auf die Wahrnehmung einer Dienstleistung durch Kunden und Mitarbeiter schon früh von Bitner (1992) und Wagner (2000) beschrie-

\section{Abb. 3: Formale Erfassung von Dienstleistungssystemen}

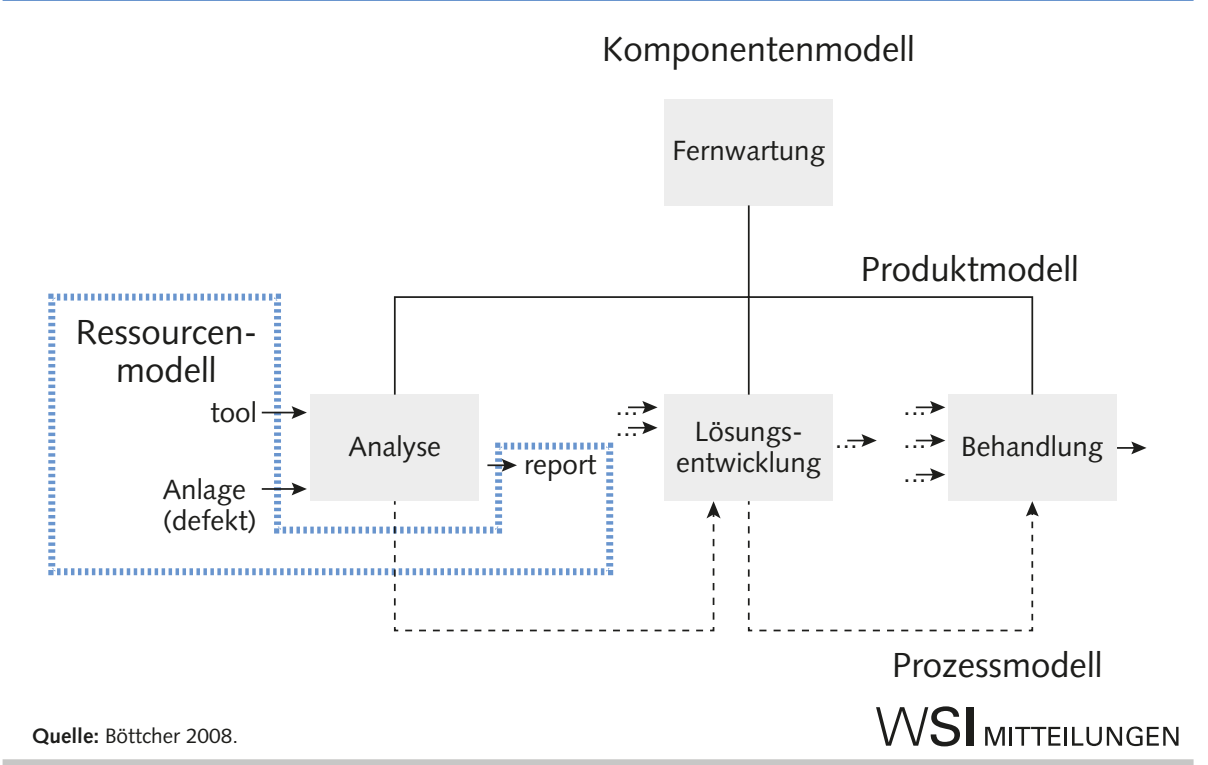

ben. Ein solches Dienstleistungsumfeld bereits in der Entwicklungsphase einer neuen Dienstleistung abzubilden, etwa um die Dienstleistung vor der Markteinführung $\mathrm{zu}$ testen, ist oft mit hohem Kosten- und Zeitaufwand verbunden, vor allem, wenn dadurch bereits existierende Dienstleistungen gestört würden (z. B. bei Verkaufsräumen und Filialen, in denen gleichzeitig mehrere Dienstleistungen angeboten werden) oder die Umgebung für die neue Dienstleistung erst noch geschaffen werden muss (z.B. Neubau von Gebäuden). Allerdings können Ansätze der „Virtuellen Realität" eine interessante Alternative sein, denn sie werden bereits seit mehreren Jahren erfolgreich in der Produktentwicklung und Architektur eingesetzt. Mit Hilfe von Virtueller Realität lässt sich computerunterstützt eine künstliche Welt generieren, in der die Nutzer in Echtzeit interagieren können. Mittlerweile sind die zugrundeliegenden 3D-Technologien soweit fortgeschritten, dass sehr realistische Abbilder erstellt und intuitiv bedient werden können (Spath et al. 2008).

Virtuelle Realität ist jedoch noch nicht ausreichend geeignet für eine realistische Darstellung von Personen, wie z. B. Kunden und Mitarbeitern. In den letzten Jahren werden zwar sogenannte Avatare verwendet, aber diese wirken nicht lebensecht und können nicht die emotionalen Erlebnisse vermitteln, die häufig mit Dienstleistungen einhergehen. Da dies nur mit realen Menschen möglich erscheint, lässt sich eine Simulation des Dienstleistungsumfeldes durch Virtuelle Realität mit Konzepten des "Service Theater" kombinieren (Grove et al. 2000; Grove/Fisk 1992). Hierbei werden Elemente aus der Welt des Theaters genutzt und Dienstleistungen werden ähnlich inszeniert wie eine Theateraufführung, d.h. es gibt im übertragenen Sinne Drehbücher, Rollen und Bühnenbilder. Die Akteure einer Dienstleistung sind dabei entweder reale Kunden und Mitarbeiter oder sie können alternativ durch professionelle Schauspieler dargestellt werden. Die Kombination aus Virtueller Realität und Service Theater wird am Fraunhofer IAO bereits seit 2006 erfolgreich genutzt, um neue Dienstleistungen zu visualisieren und zu simulieren (Fraunhofer IAO 2008). Es zeigt sich, dass die Verknüpfung aus Engineering-Know-how mit Ideen aus der Dienstleistungsforschung zu interessanten neuen Ansätzen für die Realisierung neuer Dienstleistungen führen kann.

\section{Weitere Entwicklungs-
perspektiven}

Seit den 1990er Jahren hat sich die Auseinandersetzung in Politik, Wirtschaft und Gesellschaft mit dem Thema Dienstleistungsinnovation deutlich intensiviert. Ursprünglich nur wenig mehr als ein Schlagwort hat sich Service Engineering seitdem zu einem anerkannten Bereich der Dienstleistungsforschung entwickelt. Inzwischen 


\section{Abb. 4: Perspektiven des Service Engineering}

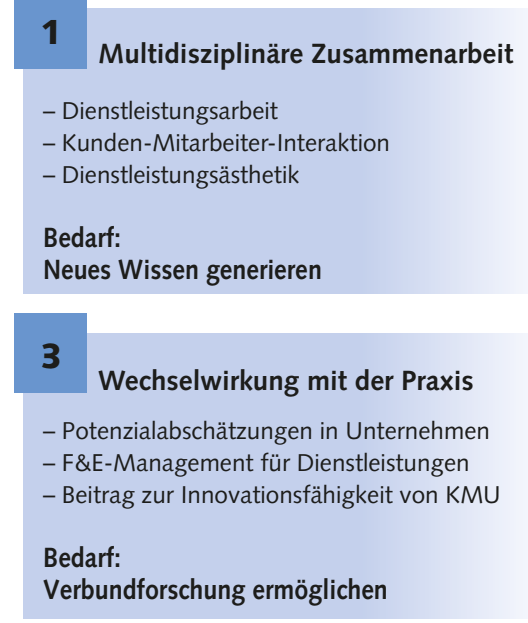

Quelle: Fraunhofer IAO 2011
2 Nutzung von Engineering-Know-how

- Service Prototyping

- Simulieren und Testen von Dienstleistungen

- Verzahnung von Technologie- und Dienstleistungsentwicklung

Bedarf:

Ingenieurwissenschaften verstärkt einbinden

4

Aufbau internationaler Netzwerke

- Schärfung des deutschen Forschungsprofils

- Aufbau einer internationalen Community

- Ausbau der internationalen Führungsposition

Bedarf:

Plattformen schaffen können wir auch international beobachten, dass dieser Ansatz auf Interesse stößt. Es lässt sich festhalten, dass in vergleichsweise kurzer Zeit ein solides Fundament geschaffen wurde, auf dem künftige Forschungs- und Entwicklungsarbeiten aufsetzen können. Für die weitere Entwicklung dieses Ansatzes sollte beispielsweise die multidisziplinäre Zusammenarbeit, die weitere Nutzung von Engineering-Knowhow, die Wechselwirkung mit der Praxis und der Aufbau internationaler Netzwerke verstärkt werden (vgl. Abbildung 4).

Durch die Intensivierung der multidisziplinären Zusammenarbeit lassen sich neue Erkenntnisse für die Entwicklung und Gestaltung von Dienstleistungen gewinnen. Von Interesse sind hierbei beispielsweise auch neue Gestaltungskonzepte von Dienstleistungsarbeit, die dem Anspruch „Guter Arbeit“ gerecht werden. Handlungsbedarf scheint es diesbezüglich reichlich zu geben (ver.di 2011). So könnten beispielsweise arbeitswissenschaftliche Erkenntnisse (Wissen zur Arbeitsplatz- und Arbeitssystemgestaltung) bereits bei der Entwicklung neuer Dienstleistungen genutzt werden. Aber auch umgekehrt könnten vermutlich die dienstleistungsspezifischen Herausforderungen die Arbeitswissenschaft inspirieren. Weitere interessante Herausforderungen für eine verstärkte $\mathrm{Zu}$ sammenarbeit verschiedener Disziplinen liegen in der Beschäftigung mit Fragen der Kunden-Mitarbeiter-Interaktion (z. B. Skripttechniken, Service Theater) und in der gezielten Ausgestaltung der emotionalen Komponenten von Dienstleistungen. In dem 2010 veröffentlichten Positionspapier der Initiative „Social Science Service Research "finden sich auch Forschungsbedarfe, die für die Weiterentwicklung des Service-Engineering-Ansatzes bedeutsam sind (Initiative „Social Science Service Research“2010).

Ein weiterer Impuls für zukünftige Arbeiten zur systematischen Entwicklung von Dienstleistungen dürfte in der verstärkten Nutzung ingenieurwissenschaftlichen Know-hows liegen. Ein Beispiel ist die Anpassung von Vorgehensmodellen zur schnellen Produktentwicklung für den Dienstleistungsbereich - quasi im Sinne eines „Service Prototyping“. Gerade angesichts des zunehmenden Wettbewerbsdrucks auf vielen Dienstleistungsmärkten scheint sich hier ein steigender Bedarf in der Praxis herauszukristallisieren. Der Einsatz eines Service Prototyping wird jedoch nur möglich sein, wenn es stärker als bisher gelingt, Dienstleistungskonzepte bereits in einer sehr frühen Entwicklungsphase zu simulieren und zu testen, um die jeweiligen Prototypen anschließend sukzessiv bis zur Implementierung und Markteinführung zu vervollkommnen. Auf einer technischen Ebene gewinnen Fragen der Modellierung und Formalisierung an Bedeutung (Kern et al. 2009; Böttcher 2008). Auf Basis dieser Arbeiten wird es zukünftig möglich sein, komplexe Dienstleistungsprodukte als Produktfamilien mit Hilfe von Leistungsplattformen und Konfigura- toren zu beschreiben und zu managen, was in der Zukunft ein umfassendes technisch unterstütztes Service Lifecycle Management erahnen lässt. Gleichzeitig werden durch diese Bestrebungen Qualitäts- und Produktivitätsanforderungen mess- und steuerbar sowie eine Standardisierung von Leistungselementen in bestimmten Branchen möglich (vgl. z. B. DIN SPEC 1199 für Remote Services).

Hilfreiche Anregungen für die Weiterentwicklung dieses systematischen Entwicklungsansatzes kommen zudem aus der Praxis. Als angewandte Fachdisziplin profitiert Service Engineering in vielfältiger Weise aus der Zusammenarbeit zwischen Forschungseinrichtungen und Unternehmen. So lassen sich Forschungsarbeiten schon zu Beginn an den Anforderungen der Praxis ausrichten und innovative Firmen bilden wiederum eine wichtige Plattform zur Erprobung neuer Konzepte. Aus Praxissicht interessieren dabei vor allem folgende Fragen: Wann lohnt sich die systematische Vorgehensweise zur Entwicklung von Dienstleistungen für Unternehmen (Potenzialabschätzung)? Welche organisatorischen Voraussetzungen sind zur kontinuierlichen Entwicklung neuer Dienstleistungen erforderlich (F\&EManagement)? Eine geeignete Basis für die Zusammenarbeit zwischen Wissenschaft und Praxis bilden sogenannte Verbundprojekte, d.h. Forschungsvorhaben, die gemeinsam von Unternehmen und wissenschaftlichen Einrichtungen bearbeitet werden. Schließlich ist eine stärkere Verknüpfung von Dienstleistungsentwicklung und modernem Innovationsmanagementansätzen, wie beispielsweise „Open Innovation“ oder „User-driven“ Ansätzen (Meyer/Thieme 2010) notwendig.

Ein anderer Aspekt hinsichtlich der zukünftigen Entwicklung dieses Ansatzes liegt im Aufbau internationaler Netzwerke. Internationalisierung macht auch vor Forschung und Entwicklung keinen Halt. Ganz im Gegenteil - im vergangenen Jahrzehnt haben gerade hier Internationalisierungstendenzen deutlich zugenommen. Die Ursachen sind vor allem in immer kürzer werdenden Inventions- und Innovationszyklen und der damit verbundenen Notwendigkeit des schnellen und effizienten Wissensaustauschs zu sehen. Neue Informations- und Kommunikationstechnologien, beispielsweise Web 2.0-Technologien, haben den Weg für völlig neue Formen der internationalen Zusammenarbeit 
geebnet. Die Integration in internationale Forschungsnetzwerke und vor allem das internationale Ansehen von Forschung und Entwicklung sind zu Erfolgsfaktoren der Entscheidungsfindung geworden, die die Standortwahl sowohl in den Bereichen der Wirtschaft als auch der Wissenschaft mitbestimmen. Service Engineering ist ein Forschungsgebiet im Dienstleistungsbereich, das maßgeblich durch deutsche Arbeiten geprägt wurde. Auch international wird dem mittlerweile große Anerkennung gezollt: „The introduction of the customercentered approach in service systems design and modeling has been motivated by the theory of service systems engineering developed in Germany ..." (Karwowski et al. 2010).

\section{LITERATUR}

Albrecht, K./Zemke, R. (1985): Service America! Doing Business in the New Economy, Dow Jones-Irwin, Homewood

Arai, T./Shimomura, Y. (2004): "Proposal of Service CAD System - A Tool for Service Engineering", ín: CIRP Annals - Manufacturing Technology 53 (1), S. 397-400

Bienzeisler, B./Herman, S. (2010): Zurück in die Zukunft mit "Dienstleistungsfacharbeit", in: WISO Diskurs Juni, Perspektiven der Erwerbsarbeit: Facharbeit in Deutschland, hrsg. von der Friedrich-Ebert-Stiftung, S. 55-66

Bitner, M. J. (1992): Servicescapes: The Impact of Physical Surroundings on Customers and Employees, in: Journal of Marketing 56 (2) S. 57-71 Böttcher, M. (2008): Architektur integrierter Dienstleistungssysteme Konzeption, Metamodell und technikraumspezifische Konkretisierung, Universität Leipzig

Bowers, M. R. (1985): An Exploration into New Service Development: Process, Structure and Organization, Texas A\&M University, Texas Bullinger, H.-J. (Hrsg.) (1995): Dienstleistung der Zukunft, Wiesbaden Bullinger, H.-J./Fähnrich, K.-P./Meiren, T. (2003): Service engineering. Methodical development of new service products, in: International Journal of Production Economics 85 (3), S. 275-287

Bullinger, H.-J./Scheer, A.-W. (Hrsg.) (2006): Service Engineering. Entwicklung und Gestaltung innovativer Dienstleistungen, Berlin

Burger, T./Lorenz, R./Meiren, T./Neus, A./Schnalzer, K./Schulteß, P./ Schultz, C. (2010): Dienstleistungsinnovation und Methoden: Herausforderungen und Handlungsempfehlungen aus Sicht von Wissenschaft und Praxis, Projektbericht, Fraunhofer-Institut für Arbeitswirtschaft und Organisation (IAO), Stuttgart

Edvardsson, B./Gustafsson, A./Johnson, M. D./Sandén, B. (2000): New Service Development and Innovation in the New Economy, Lund Edvardsson, B./Gustafsson, A./Kristensson, P./Magnusson, P. R./ Matthing, J. (Hrsg.) (2006): Involving Customers in New Service Development, London

Edvardsson, B./Meiren, T./Schäfer, A./Witell, L. (2010): New Service Development, Studie, Universität Karlstad, Fraunhofer-Institut für Arbeitswirtschaft und Organisation (IAO), Stuttgart und Hochschule Luzern Ernst, G. (2011): Forschungs- und Innovationspolitik zu Dienstleistungen in Deutschland sowie im europäischen und internationalen Umfeld, Materialsammlung, Bonn

Fähnrich, K.-P. /Meiren, T. (2007): Service Engineering: State of the Art and Future Trends, in: Spath, D./Fähnrich, K.-P. (Hrsg.): Advances in Services Innovations, Berlin, S. 3-16

Fähnrich, K.-P./van Husen, C. (Hrsg.) (2008): Entwicklung IT-basierter Dienstleistungen. Co-Design von Software und Services mit ServCASE, Heidelberg
Fitzsimmons, J./Fitzsimmons, M. (Hrsg.) (2000): New Service Development, London

Fraunhofer-Institut für Arbeitswirtschaft und Organisation (IAO) (2008): ServLab, Film, www.youtube.com/watch?v=X6Ay3kJ-RfNM (gelesen am 10.03.2011)

Ganz, W./Meiren, T. (Hrsg) (2002): Service research today and tomorrow. Spotlight on international activities, Stuttgart

Grove, S. J./Fisk, R. P. (1992): The Service Experience as Theater, Advances in Consumer Research 19, S. 455-461

Grove, S. J./Fisk, R. P./John, J. (2000): Services as Theater: Guidelines and Implications, in: Swartz, T. A./lacobucci, D. (Hrsg.): Handbook of Services Marketing and Management, New York, S. 21-36

Hacker, W. (2009): Arbeitsgegenstand Mensch: Psychologie dialogischinteraktiver Erwerbsarbeit, Lengerich

Initiative "Social Science Service Research" (2010): Positionspapier, www.3sresearch.de (gelesen am 10.03.2011)

Karwowski, W./Salvendy, G./Ahram, T. (2010): Customer-centered design of service organizations, in: Karwowski, W./Salvendy, G. (Hrsg.): Introduction to Service Engineering, Wiley/Hoboken/New York, S. $179-206$

Kern, H./Böttcher, M./Kühne, S./Meyer, K. (2009): Ansatz zur ganzheitlichen Erstellung und Verarbeitung von Dienstleistungsmodellen, in: Oliver, T./Nüttgens, M. (Hrsg.): Dienstleistungsmodellierung, Berlin, S. $3-16$

Mager, B. (2005): Service Design - A Review, Cologne

Mandelbaum, A. (1999): Service Engineering: Modelling, Analysis and Inference of Stochastic Service Networks, Arbeitspapier, Israel Institute of Technology, Haifa

Meiren, T. (1999): Service Engineering: Systematic Development of New Services, in: Werther, W. /Takala, J./Sumanth, D. J. (Hrsg.): Productivity \& Quality Management Frontiers, Bradford, S. 329-343 Meiren, T./Barth, T. (2002): Service Engineering in Unternehmen umsetzen. Leitfaden für die Entwicklung von Dienstleistungen, Stuttgart Meyer, K./Fähnrich, K.-P./Müller, R./Freitag, M. (2008): Entwicklung internationaler produktbezogener Dienstleistungen - Ein Handlungsleitfaden für kleinere und mittlere Unternehmen, Leipziger InformatikVerbund (LIV), Leipzig

Meyer, K. (2009): Software-Service-Co-Design: Eine Methodik für die Entwicklung komponentenorientierter IT-basierter Dienstleistungen, Leipziger Informatik-Verbund LIV, Leipzig

Meyer, K./Münter, D. (2010): Workshop Human-Service-Interaction. Mensch \& Computer 2010, in: Ziegler, J./Schmidt, A.: Fachübergreifende Konferenz für interaktive und kooperative Kulturen, München, S. 393-394 
Meyer, K./Thieme, M. (2010): Activating the Innovation Potential of SME: The Bottom-Up-Approach. Informatik 2010 - Business Process and Service Science - Proceedings of ISSS and BPSC, Gesellschaft für Informatik e.V. (GI), Leipzig

Organisation for Economic Co-operation and Development (OECD) (2005): Promoting Innovation in Services, Organisation für wirtschaftliche Zusammenarbeit und Entwicklung (OECD), Paris

Ramaswamy, R. (1996): Design and Management of Service Processes, Addison-Wesley, Boston/MA.

Sakao, T./Shimomura, Y. (2006): Service Engineering: a novel engineering discipline for producers to increase value combining service and product, in: Journal of Cleaner Production 15 (6), S. 590-604

Scheer, A.-W./Spath, D. (Hrsg.) (2004): Computer Aided Service Engineering, Informationssysteme in der Dienstleistungswirtschaft, Berlin Spath, D./Bauer, W./Dangelmaier, M. (2008): Virtual Service System Engineering, in: American Society of Mechanical Engineers (ASME) (Hrsg.): Proceedings of the 9th ASME Engineering Systems Design and Analysis Conference, Israel Institute of Technology, Haifa
Spath, D./Ganz, W. (Hrsg.) (2008): The Future of Services. Trends and Perspectives, München

United Nations Economic Commission for Europe (UNECE) (2011):

Promoting Innovation in the Services Sector, New York/Genf

Vereinte Dienstleistungsgewerkschaft (ver.di) (2011): Arbeit mit Kunden, Patienten, Klienten. So bewerten Beschäftigte in den Dienstleistungs-Branchen die Arbeitsbedingungen, ver.di Reihe Arbeitsberichtserstattung aus Sicht der Beschäftigten

Wagner, J. (2000): A Model of Aesthetic Value in the Servicescape, in: Swartz, T.A./lacobucci, D. (Hrsg.): Handbook of Services Marketing and Management, New York, S. 65-88

Zühlke-Robinet, K. (2009): Brücke zum Mittelstand geschlagen, in: Dienstleistungen aus dem Labor, Karlsruhe. 